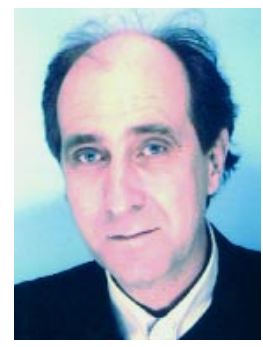

Ulrich Lamparter

\title{
Stressbedingte Aspekte des Hörsturzes
}

Gerhard Goebel und Ulrich Lamparter

Medizinisch-Psychosomatische Klinik Roseneck, Prien am Chiemsee

Poliklinik für Psychosomatische Medizin und Psychotherapie, Universitätsklinikum

Hamburg-Eppendorf

psychoneuro 2004; 30 (6): 337-341

In der Literatur finden sich belegende Hinweise, dass der Hörsturz in Zusammenhang mit Belastungen und Stressoren steht; Hörsturzpatienten insgesamt haben jedoch nicht unbedingt mehr Stress als andere Menschen und Stress führt auch nicht zwangsläufig zum Hörsturz. Die Belastungen vor dem Hörsturz erscheinen lediglich chronifizierter und kritischer, gepaart mit deutlich belastenderen Alltagssorgen. Spezifische Lebensereignisse, die für das Hörsturzgeschehen verantwortlich sein könnten, wurden nicht herausgefunden. Vielmehr legen die empirischen Befunde ein komplexeres Modell der Hörsturzentstehung unter Stress nahe: Der Hörsturz konstelliert sich vielmehr in einer komplexen biographischen Situation bei Menschen mit eher guter bis sehr guter Realitätsanpassung oft unter den Bedingungen beruflicher Belastung. Wird eine solche Belastung ungenügend wahrgenommen bzw. verdrängt und trifft sie in auslösenden Situationen auf eine biographisch bedingte innere „Resonanz“, kann es zu einem nicht mehr regulierbaren Konflikt kommen. Dabei auftretende Ohnmachtsgefühle, Wut- oder Schuldgefühle münden, da nur unvollkommen integriert, in eine „vegetativen Entgleisung“ mit der Folge Hörsturz. Persönlichkeitsmerkmale, wie sie beim Hörsturz gefunden werden, sind unspezifisch, ähnliche Charakteristika finden sich bei Hochdruckpatienten (Aggressionshemmung), Blutspendern (soziale Angepasstheit, Moralität), Kopfschmerz- und Migränepatienten (Perfektionismus, hoher Anspruch an sich selbst), sie bereiten jedoch den Boden für die psychosomatische Reaktion. Speziell für den Hörsturz ist am ehesten eine vermehrte Sensibilität anzunehmen. Schreck, z.B. bei Feuersgefahr oder durch plötzlich hereingebrochenen tiefen Kummer von temporären oder bleibendem Ohrensausen oder von Schwerhörigkeit befallen wurden. Es ist dies erklärlich, wenn man bedenkt, dass die Gemüthsaffecte zu den stärksten, auf die Gefässnerven (die Vasoconstrictoren und Dilatatoren) wirkenden Reizen zählen.“

\section{Klinik}

Wegen seines plötzlichen („Sturz“) und unerwartenden Auftretens ist der Hörsturz für viele Betroffene mit Erschrecken und Unsicherheit, längerfristig auch mit Angst vor einer unerklärlichen Erkrankung und weiterer Verschlechterung verbunden. Am Anfang verspüren die Betroffenen die plötzlich einsetzende Hörminderung („wie Watte“), die innerhalb von Sekun-

den oder Minuten aus völligem Wohlbefinden heraus eintreten kann. Gelegentlich geht dem Hörsturz ein Druckgefühl im Ohr voraus oder in seltenen Fällen wird ein Schwank- oder Drehschwindel angegeben. In auffallend vielen Fällen wird der in der Regel einseitig auftretende Hörsturz beim morgendlichen Aufwachen bemerkt. Tinnitus ist entgegen landläufiger Meinungen nie der Vorbote eines Hörsturz, in $60 \%$ der Fälle jedoch ein Folgesymptom, das sich innerhalb weniger Stunden bis Tagen einstellt. Oft bemerken die Patienten den Tinnitus im Zusammenhang mit der Gehörerholung. Ein ausbleibender Tinnitus ist bezüglich der Gehörerholung eine prognostisch eher ungünstige Konstellation (1).
Nach heutigem medizinischen Verständnis wird Hörsturz nicht als eigenständige Krankheit, sondern als Symptom unklarer Genese (Ursache) verstanden. Es handelt sich um eine partielle, selten komplette, Funktionsstörung des Innenohrs. Auch ohne Behandlung erholt sich das Gehör in 25 bis $75 \%$ der Fälle, wobei eine Schädigung im Tieftonbereich sich häufiger spontan zurückbildet. Des weiteren ist die Spontanerholungsrate um so günstiger, je jünger die Patienten sind und je besser der Ausgangszustand des entsprechenden Gehörs ist. Definitionsgemäß ist als Ursache ein Akustikusneurinom, der Menière-Anfall, Ruptur des runden Fensters, BaroTrauma, Otosklerose, Periarteriitis nodosa, psychogene Hörstörung, 
u.a. auszuschließen. Erst wenn keine plausiblen Erklärungen für die Funktionsstörung des Innenohrs gefunden werden, wird man korrekter Weise von einem idiopathischen Hörsturz sprechen können.

\section{Häufigkeit}

Die Forschung zum Hörsturz hat erst richtig eingesetzt, als durch Erweiterung der HNO-Diagnostik einschließlich Einführung der Audiometrie der Hörsturz besser von der psychogenen Hörstörung abgegrenzt werden konnte. So wurden noch im ersten Weltkrieg Soldaten, die im Geschützdonner vorübergehend ertaubten, als „Kriegsneurose des Ohres“ verkannt!

Der Hörsturz zählt zu den zunehmenden Krankheitsbildern mit schätzungsweise 10 bis 35 neuen Fällen auf 100000 Einwohner pro Jahr (13). Für Deutschland müsste demnach eine jährliche Inzidenzrate von etwa 15000 Neuerkrankungen angenommen werden. Es muss allerdings von einer hohen Dunkelziffer ausgegangen werden. Nach klinischen Studien treten etwa 60\% der Hörstürze zwischen dem 30. und 60. Lebensjahr auf (mit einem mittleren Altersdurchschnitt von etwa 46 Jahren). Aber auch Kinder können von Hörsturz betroffen sein (16). Die Hörsturzhäufigkeit verteilt sich zwischen Männer und Frauen ähnlich. Unterschiede zwischen ländlichen und städtischen Regionen sind nicht bekannt. Nach dem Erstauftreten eines Hörsturz muss in knapp 10\% der Fälle mit einem Rezidiv gerechnet werden. Befragt man Berufsgruppen nach Hörsturz, so scheinen akademische Berufe (Universitätsprofessoren) mit einer Lebenszeitprävalenz von 5\% am häufigsten betroffen zu sein (3).

\section{Stressbedingte Aspekte}

Der Begriff „Stress“ geht u.a. auf den kanadischen Biologen Dr. Hans Selye zurück, der ihn in den 50erJahren in die Medizin einführte. Er definiert damit eine „nichtspezifische Reaktion des Körpers auf jede an ihn gerichtete Anforderung" wie Not, Druck, Anspannung. Stress ist somit die körperliche Antwort des Organismus auf bedrohliche oder herausfordernde Situationen, mit deren Hilfe die zu ihrer Bewältigung notwendige Energie bereitgestellt wird. Von ihm stammen auch die Begriffe „Eu-Stress“ (für positiven Stress) und „Dis-Stress“ (für negativen Stress). Kennzeichen des EuStress ist ein positives Gefühl der Herausforderung, die bereitgestellte Energie wird in der Regel verbraucht. Dis-Stress wird als bedrohlich erlebt, die bereitgestellte Energie wird meist nicht abgebaut.

Neben biologischen bzw. medizinischen Variablen wird für die Erklärung des Hörsturz von Klinikern und Psychotherapeuten gleichermaßen häufig Stress bzw. emotionale Belastung als bedeutender ätiologischer Faktor genannt. Auch die Betroffenen sehen dies ähnlich: So finden sich in Statistiken von Patienten psychosomatischer Kliniken mit dekompensiertem chronischen Tinnitus bis zu 53\% Patienten, deren Tinnitusursache auf einen Hörsturz zurückgeführt wird $(6,7)$. Diese von den Prävalenzdaten extrem abweichende Häufung erklärt sich durch das Verständnis der Hörsturzbetroffenen, die eine Psychotherapie als Rezidivprophylaxe eines Hörsturz ansehen und entsprechend erwartungsvoll in eine psychotherapeutischen Behandlung kommen (8).

Es existieren eine Reihe von Arbeiten zu psychischen Aspekten bei Hörsturz, wobei die Ergebnisse von ca. 14 Arbeitsgruppen als wissenschaftlich akzeptabel erscheinen. Insgesamt wurden damit unterschiedliche psychische Bereiche bei mehr als 850 Hörsturz-Patienten empirisch untersucht. Neuere Arbeiten konnten ihre Befunde mit Kontroll- und Vergleichsgruppen absichern. Die eingesetzten psychometrischen Testverfahren sind weitgehend valide.

\section{Zusammenhänge mit Lebensbelastungen}

Beobachtungen über psychosomatische Zusammenhänge, wie sie Fowler 1950 (4) erstmals systematisch zusammenstellte, wurden durch zahlreiche empirische Untersuchungen bestätigt. Globale Befragungen (z.B. „Hatten Sie psychischen Stress?“) zeigten je nach Untersu- chern allerdings unterschiedlich stark variierende Ergebnisse.

Methodische Probleme ergeben sich wie bei allen ähnlich gelagerten Studien dadurch, dass Stressfaktoren durch die Betroffenen eventuell erst im Nachhinein als Erklärungsmodell angesehen werden, da andere Erklärungen fehlen und es einem weitverbreiteten Bedürfnis des Menschen entspricht, Erklärungen für Schicksalsereignisse zu finden. Auch sind sich wandelnde Weltbilder Einflussgrößen für solche Bereiche: Bei Fowler (4) gaben nur etwa $10 \%$ der Betroffenen von sich aus psychische Auslöser an, gegenüber den Patienten von Kropp \& v. Rad (11) bzw. Lamparter (12), von denen über $70 \%$ spontan „Stress“ als Hörsturzauslöser nannten.

\section{Zusammenhänge mit individuellen Aspekten}

Oft fällt den behandelnden Ärzten auf, dass viele Patienten mit Hörsturz sich als besonders sozial engagiert darstellen und z.B. sich in Lehrberufen besonders häufen (3). So wurde bei der Suche nach biographischen Besonderheiten, Persönlichkeitsmerkmalen und besonderen Lebensbewältigungsstilen von der modellhaften Vermutung (Hypothese) ausgegangen, dass solche Faktoren z.B. einen in einer bestimmten Weise defizitären Lebensstil bedingen, der dann im klassischen Sinn der Psychosomatik eine vegetative Fehlsteuerung mit körperlicher Folge (Hörsturz) verursacht.

Erste systematische Befunde zu Persönlichkeitsmerkmalen wurden von Dohse et al. (2) bei 30 HörsturzPatienten im Vergleich zu 35 Otosklerose-Patienten erhoben. Die Hörsturzbetroffenen erschienen vermehrt nervös und weniger selbstbewusst. Einzelheiten zur Stichprobe, Untersuchungsvorgehen und Statistik sind unklar; es werden ohne Mittelwertsangaben Abweichungen gegenüber Normstichproben in einigen Skalen des Freiburger Persönlichkeitsinventar (FPI-R) berichtet.

Neusser \& Knoop (14) fokussierten in Ihrer Untersuchung bei 56 Akut-Hörsturzpatienten die lebensverändernden Ereignisse und Per- 
sönlichkeitsmerkmale (1 Wochenfenster, keine Angaben zum Hörtest) im Vergleich zu Krankenhauspersonal. Ausgeschlossen wurden Patienten, bei denen die Infusionstherapie keine rasche Erholung des Gehörs bewirkte. Es fanden sich mehr belastende und anhaltende Lebensereignisse sowie erhöhte Werte für Depression und Hysterie (MMPI), die sich insgesamt jedoch im Bereich der Durchschnittsbevölkerung bewegten.

Kropp \& von Rad (11) beobachteten in einer explorativen Untersuchung (Non-direktives Gespräch) von 64 Hörsturzpatienten mit 90 Hörstürzen in 59 von 90 Fällen keine spezifischen Persönlichkeitsmerkmale. Die Patienten berichteten „von sich aus über einen zeitlichen $\mathrm{Zu}$ sammenhang" zwischen starker psychischer Belastung und Hörsturz. Die Untersucher selbst sahen nur in sieben Fällen einen solchen Zusammenhang als gegeben an, in den restlichen 24 Fällen verwarfen sie einen derartigen Zusammenhang.

Ein größerer Datensatz zur Biographie von Hörsturz-Patienten ist der Promotionsarbeit von Hoffmeister (9) aus Tübingen zu entnehmen. Hier wurden 58 Patienten mit akutem Hörsturz im Vergleich zu einer Gruppe von Menière-Patienten, Migräne-Patienten, Kopfschmerz-Patienten und einer Gruppe von gesunden Personen mit einem Fragebogen zur Biographie untersucht.

Am unauffälligsten stellten sich die Hörsturz-Patienten dar: Sie schilderten ihre Ursprungsfamilie als sehr ideal, es gab wenig Streitigkeiten zwischen den Eltern im Gegensatz zu den sehr negativ urteilenden Kopfschmerz-Patienten. Bis auf eine etwas unpünktliche und weniger fürsorgliche Mutter wurden die Eltern als ideale Personen beschrieben. Der Erziehungsstil war bei den Hörsturz-Patienten am demokratischsten und es wurden wenige Schulprobleme erinnert. Auch die aktuelle Partnerschaft wurde im Gegensatz zu den anderen Vergleichs- und Kontrollgruppen als besonders zufriedenstellend beschrieben. Sie schätzten sich pünktlich, ordentlich und hilfsbereit im Sinne einer Überangepasstheit ein. Erst auf den zweiten Blick fiel ein extrem sozial erwünschtes Verhalten auf, das heißt, Hörsturzbetroffene scheinen vor anderen und vielleicht auch vor sich selbst ihre Situation im Sinne gesellschaftlicher Erwartungen idealisierend darzustellen.

Eine weitere umfangreiche Untersuchung zu psychischen Aspekten stammt von Lamparter (12). Er explorierte mit einem psychoanalytischen Vorgehen und einer systematisierten Befunderhebung sowie einer wissenschaftlich sehr aufwändigen Testbatterie 50 Patienten mit frischem Hörsturz (10 Tagesfenster) und untersuchte sie fünf Jahre später nochmals (Rücklaufquote über $70 \%$ ) sowie einen Pool von 160 Patienten, deren Hörsturzereignis unterschiedlich lange zurücklag. Auch hier erschienen die Hörsturz-Patienten bezüglich ihrer Persönlichkeit wenig auffällig und psychisch deutlich gesünder als Patienten psychosomatischer Ambulanzen. Lamparter unterschied verschiedene psychosomatische Funktionen des Hörsturzes und fand psychodynamisch häufig Gewissenskonflikte und eine oft unbewusste ohnmächtige Pro-

\section{Abb. 1 Stress-Hörsturzmodell (nach 12)}

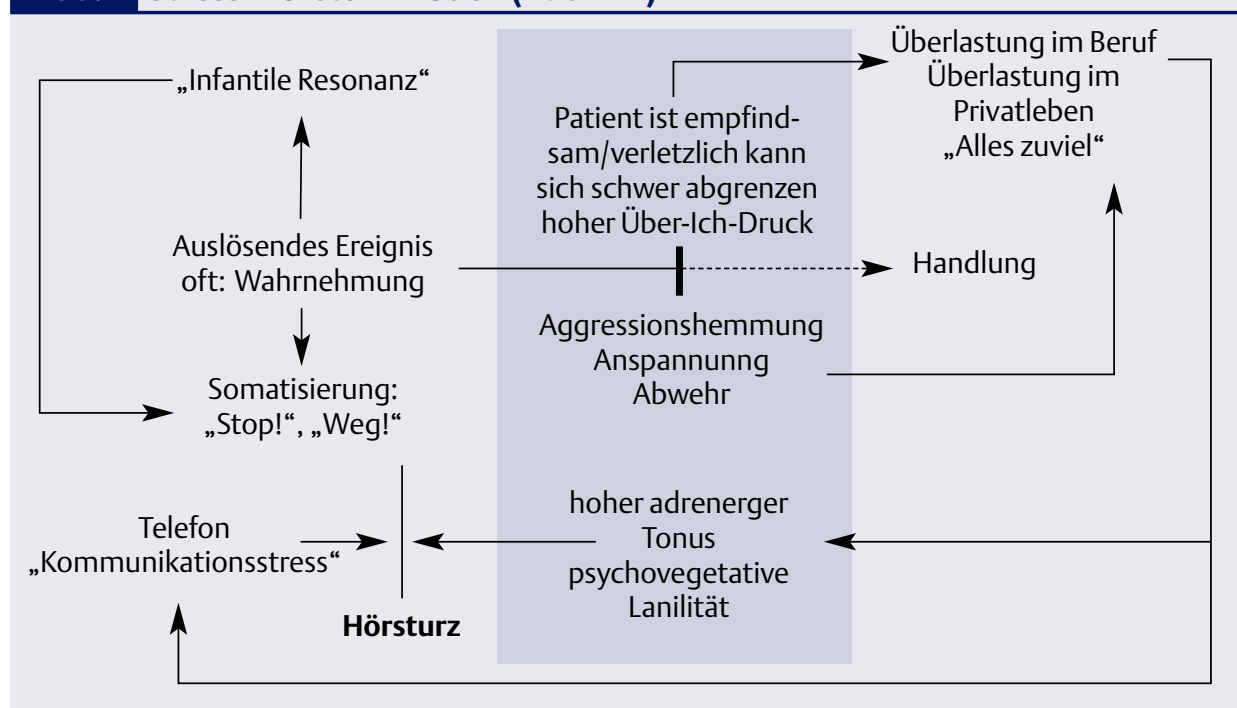

Die aus einer (akustischen) Wahrnehmung eines Ereignisses (auslösende Situation) folgende Handlung wird blockiert und über eine Somatisierung abgewehrt. Berufliche und private Überlastung, insbesondere „Telefonstress“ tragen zur Somatisierung bei, indem sie die psychischen Anpassungsressourcen des Individuums erschöpfen und disponierende somatische Faktoren bahnen: Es ist „alles zuviel“. Individuelle persönlichkeitsspezifische Faktoren bedingen die Handlungshemmung, aber auch die Tendenz sich zu überlasten. Eine „infantile Resonanz“(mit Konfliktmustern der Kindheit) gibt der auslösenden Situation besondere Wirkkraft testhaltung unter den Hörsturzbetroffenen. Als typische primärpersönliche Züge fand er eine hohe Sensibilität, stark entwickeltes Pflichtgefuhl, Aggressionshemmung sowie Schwierigkeiten sich abzugrenzen (Abb. 1). Charakteristika fanden sich in ähnlicher Weise auch bei einer VerHörsturz-Patienten und Blutspender ăhneln sich offenbar in ihrer Morat! In der relativ einseitigen Stichprobe (es befanden sich kaum Arbeigruppe), fiel eine hohe Identifikation mit dem Beruf und Akzeptanz der damit einhergehenden Belastung auf. Vor allem die beruflich Belasteten konnten sich weniger ,abschotten": Unter den Patienten fanen sich häufig Berufe, die als „Komikationsberufe" charakterisiert Arden können: Es waren Pfarrer, Arzte, Psychotherapeuten, Lehrer Kindergärtnerinnen, Zeitungsweretc. In gewisser Weise passe ergebnisse einer Fragebogenaktion bezüglich Hörsturz und Tinnitus von Fleischer et al. (3) bei gleichsgruppe von Blutspendern: 
unterschiedlichen Berufsgruppen: Die höchste Häufigkeit von Hörstürzen $(5 \%)$ wurde bei den 1.600 hessischen Hochschulprofessoren gefunden (Rücklaufquote etwa 60\%) im Gegensatz zu einer Hörsturzhäufigkeit von $3 \%$ bei den befragten Soldaten und weniger als $1 \%$ bei den befragten Jugendlichen. 12\% der Hochschulprofessoren in Hessen gaben chronische Ohrgeräusche an, die für sie allerdings wenig belastend waren, $60 \%$ blieben auch nach dem Hörsturz „tinnitusfrei“.

Bei der empirischen Überprüfung des Stressbewältigungsverhaltens fanden Kröger et al. (10) bei einer Stichprobe von 50 Hörsturz-Betroffenen der TU Aachen im Wesentlichen die Beobachtungen von Neuser (14) und Kropp \& v. Rad (11) bestätigt: Die Untersucher eruierten eine unterdurchschnittliche Selbstaufmerksamkeit sowie ungenügende Bewältigungsstrategien. Hörsturz-Patienten scheinen in Krisensituationen offenbar weniger mit intra- und interpersonellen Lösungsstrategien zu reagieren, sondern sich mehr mit körperlichen Missempfindungen bis hin zu einem Hörsturzrezidiv zu äußern (Somatisierung).

In einer Untersuchung von Schmitt et al. (17) wurden 40 Patienten mit akutem Tinnitus- und Hörsturz (10 Tagesfenster) bezüglich ihrer prämorbiden Stressoren, Alltagssorgen, Worrying (sich sorgen, grübeln), Stressbewältigung etc. mit konservativ behandelten HNO-Patienten verglichen. Es fanden sich für alle Bereiche klinisch relevante Unterschiede. Lediglich für die Bereiche soziale Unterstützung bzw. psychosoziale Belastung schienen keine Zusammenhänge mit dem HörsturzTinnitusauslöser zu bestehen (siehe Abb. 2).

In einer differenzierten Datenanalyse untersuchten Gerhards et al. (5) die prämorbide Stressbelastung von 21 akuten Tinnitus- und Hörsturzpatienten (3 Wochen-Fenster; $81 \%$ sekundärer Tinnitus): Es fanden sich im Vergleich zu einer teils konservativ, teils operativ behandelten HNO-Patientengruppe ohne Tinnitus weder abweichende Aspekte der Arbeitsbelastung, arbeitsbezogener Anspannung/Nervosität, Stressanfälligkeit- bzw. -reagibilität, noch chronische Stressbelastung oder eine erhöhte Belastung durch kritische Lebensereignisse. Auch beim Vergleich der Tinnitus-/Hörsturzbetroffenen, die ihre Symptomatik auf erhöhte Stressoren zurückführten (52\%) mit den Patienten, die die Fragen nach Stressauslöser ihres Tinnitus/Hörsturz verneinten, ließ sich mit den objektiven Fragebögen keine unterschiedlichen Stressniveaus belegen. Die Autoren diskutieren ihre von Hoffmeister (9), Neuser \& Knoop (14) sowie Schmitt et al. (17) abweichenden Befunde auch unter dem Aspekt, dass Unterschiede in den Merkmalen der verschiedenen Stichproben eine Erklärung der Diskrepanz sein könnten. Sie fordern weiterhin dezidiert, die Stress-Hypothese bei Hörsturz weiter zu beforschen.

\section{Abb. 2 Stress-Hörsturz-Modell (nach 17)}

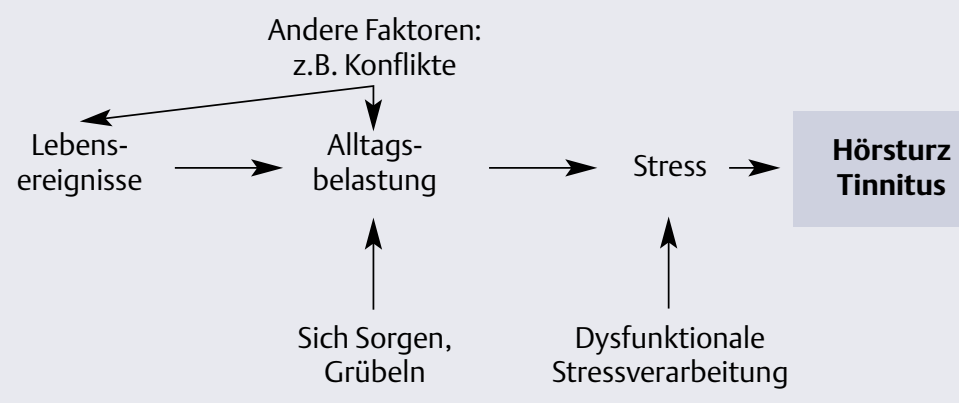

Biographische und aktuelle Konflikte sowie Sorgen und Grübeln sind entscheidende Wirkfaktoren auf die Alltagsbelastungen. Eine damit verbundene dysfunktionale Stressverarbeitung gilt letztlich als Risikofaktor von Hörsturz und Tinnitus

\section{Zusammenfassung}

Die meisten der vorgestellten $\mathrm{Ar}$ beiten finden deutliche belegende Hinweise, dass der Hörsturz in $\mathrm{Zu}-$ sammenhang mit Belastungen und Stressoren steht; Hörsturzpatienten insgesamt haben jedoch nicht unbedingt mehr Stress als andere Menschen und Stress führt auch nicht zwangsläufig zum Hörsturz. Die Belastungen vor dem Hörsturz erscheinen lediglich chronifizierter und kritischer, gepaart mit deutlich belastenderen Alltagssorgen. Spezifische Lebensereignisse, die für das Hörsturzgeschehen verantwortlich sein könnten, wurden nicht herausgefunden. Vielmehr legen die empirischen Befunde ein komplexeres Modell der Hörsturzentstehung unter Stress nahe: Der Hörsturz konstelliert vielmehr in einer komplexen biographischen Situation bei Menschen mit eher guter bis sehr guter Realitätsanpassung oft unter den Bedingungen beruflicher Belastung. Wird eine solche Belastung ungenügend wahrgenommen bzw. verdrängt und trifft sie in auslösenden Situationen auf eine biographisch bedingte innere „Resonanz“, kann es zu einem nicht mehr regulierbaren Konflikt kommen. Dabei auftretende Ohnmachtsgefühle, Wut- oder Schuldgefühle münden, da nur unvollkommen integriert, in eine „vegetative Entgleisung“ mit der Folge Hörsturz. Persönlichkeitsmerkmale, wie sie beim Hörsturz gefunden werden, sind unspezifisch, ähnliche Charakteristika finden sich bei Hochdruckpatienten (Aggressionshemmung), Blutspendern (soziale Angepasstheit, Moralität), Kopfschmerz- und Migränepatienten (Perfektionismus, hoher Anspruch an sich selbst), sie bereiten jedoch den Boden für die psychosomatische Reaktion. Speziell für den Hörsturz ist am ehesten eine vermehrte Sensibilität anzunehmen.

Bei Lamparter (12) war diese Persönlichkeitseigenschaft zusammen mit dem Gefühl „alles zu viel, ich kann es nicht mehr hören" mit einem erhöhten Rezidivrisiko verbunden (zweiter Hörsturz) und eine testpsychologisch gefundene mehr nach innen gerichtete Aggression mit einem quälenden Charakter des 
chronischen Ohrgeräusches in der Folge des Hörsturzes (5 Jahres-Katamnese). Bei einer Nachuntersuchung der Stichprobe von Schmitt et al. (17) war von 33 bisher erfassten Patienten nach drei Monaten bei etwa der Hälfte der Tinnitus chronifiziert. Bei einer ersten Analyse erschienen das aktuelle Befinden im Anfangsstadium, negative Stressbewältigungsstrategien, eine fatalistische Kontrollüberzeugung sowie die Beeinträchtigung durch den Hörverlust bzw. den Tinnitus negative Prädiktoren für die Chronifizierung.

Ein Rezidivrisiko bis 40\% (12) und dekompensierter Tinnitus nach Hörsturz implizieren daher eine psychotherapeutische Konsequenz. Die Untersuchungen und Erfahrungen der Autoren belegen eine hohe Therapiemotivation der Hörsturzpatienten mit Tinnitus. Nicht zuletzt verstehen Hörsturzpatienten Psychotherapie auch als Hörsturzprophylaxe. Sie stellen in der Klinik Roseneck die Patientengruppe mit der höchsten Erfolgsquote, zumindest bezüglich Abnahme ihrer Tinnitusbelastung, dar. Wie bereits v. Rad (15) und Gerhards et al. (5) ausführen, erweist sich der Hörsturz wie aus somatischer Sicht auch aus psychosomatischer nicht als einheitliche Erkrankung, was weitere Forschungsarbeit erfordert.

\section{Stress and sudden hearing loss}

The literature on sudden hearing loss suggests that this problem is correlated with various types of stress. However, patients with sudden hearing loss overall do not exhibit more signs of stress than other individuals, and stress itself does not inevitably lead to sudden hearing loss. On the other hand, the stress factors preceding sudden hearing loss appear to be more chronic, more salient and are compounded by more troublesome daily hassles. Pertinent research was not able to identify any specific life events that would be correlated with or causally related to sudden hearing loss. However, empirical research suggests a more complex stress model for the time before the first occurrence of the problem: sudden hearing loss afflicts individuals with good or excellent reality adaptation who experience a complex biographical situation, often in combination with difficult working conditions. If the person fails to recognize the psychological nature of these stress factors at work while experiencing at the same time major private problems, i.e. if problems at work "resonate" with private issues, the existing inner conflict is exacerbated and cannot be regulated appropriately any more. Feelings of helplessness, rage or guilt are not integrated properly and lead to a dysregulation of the vegetative nervous system with the symptom of sudden hearing loss. These patients exhibit certain nonspecific personality characteristics that, on the one hand, prepare the ground for a psychosomatic reaction, but, on the other hand, can also be found in other patient groups, e.g. in patients with high blood pressure (inhibition of aggressive impulses), individuals who donate blood (social over-adjustment, moralism), patients with headaches and migraine (perfectionism, high self-standards). Only an increased level of sensitivity seems to be specific to individuals with sudden hearing loss.

\section{Keywords:}

sudden hearing loss - stress - daily hassles - biography - psychosomatic reaction

\section{Literatur}

1. Danino J, Joachims AZ, Eliacher I. Tinnitus as a prognostic factor in sudden deafness. Am J Otolaryngol 1984; 5; 394- 396

2. Dohse J, Lehrl S, Berg M. Personality system and sudden deafness: a comparative psychological study. Adv. Otolaryngol 1981; 27: 110-113

3. Fleischer G, Hoffmann E, Lang R, Müller R. Tinnitus und Hörsturz bei Universitätsprofessoren und einigen anderen Berufsgruppen. Umweltmedizin in Forschung und Praxis 1999; 4: 144-152

4. Fowler E P. Sudden deafness. Annals Otology (St. Louis) 1950; 59: 980-987

5. Gerhards F, Schwerdfeger FP, Etzkorn M, Hasselmayer A. Psychosozialer Stress: Ein bedeutsamer ätiologischer Faktor bei Tinnitus? In: Dohrenbusch R. \& Kaspers F (Hrsg.). Fortschritte der Klinischen Psychologie und Verhaltensmedizin. Lengrich, Papst; 2001:122-135

6. Goebel G, Hiller W, Frühauf K, Fichter M. Effects of inpatient multimodal bahavioural treatment on complex chronic tinnitus-A controlled one year follow-up-study. In: Aran JM \& Dauman R. (ed.). Proceedings of the Fourth International Tinnitus Seminar. Amsterdam/New York, Kugler Publications, 1992: 465-470

7. Goebel G, Hiller W, Rief W, Fichter M. Integratives verhaltensmedizinisches stationäres Behandlungskonzept beim Tinnitus. In: Goebel G (Hrsg.). Ohrgeräusche. Psychosomatische Aspekte des chronischen Tinnitus. München, Urban \& Vogel, 2001: 139-176

8. Hiller W. \& Goebel G. Hals-Nasen-Ohren-Erkrankungen (HNO). In: Flor H. Hahlweg K. \& Birbaumer N. (Hrsg.). Enzyklopädie der Psychologie, Themenbereich B Praxisgebiete, Serie II, Klinische Psychologie; Band 4: Anwendungen der Verhaltensmedizin. Göttingen Hogrefe, Verlag für Psychologie, 2001:147-200

9. Hoffmeister K. Verhaltensmedizinische Untersuchungen zum Hörsturz. Europäische Hochschulschriften, Reihe 6: Psychologie, Bd. 255. Frankfurt, Peter Lang Verlag, 1987

10. Kröger F, Lamprecht J, Wälte D. Stressverarbeitung und Selbstaufmerksamkeit beim idiopathischen Hörsturz. Psychother. med. Psychol 1996; 46: 298-303

11. Kropp UAK \& v Rad M. (Psychosomatische Aspekte des Hörsturz. Psychother med Psychol 1988; 38: 407-412

12. Lamparter U. Studien zur Psychosomatik des Hörsturz. Habilitationsarbeit. Hamburg, Medizinische Fakultät der Universität Hamburg,1994

13. Michel O. Der Hörsturz. Stuttgart, Georg Thieme Verlag, 1998

14. Neuser ] \& Knoop T. Sudden idiopathic hearing loss: Psychopathology and antecedent stressfull lifeevents. British Journal of Medicin Psychology 1986; 59: 245-251

15. von Rad M. Hörsturz. In: Bräutigam W, Christian P, v Rad M. (Hrsg.). Psychosomatische Medizin. Eine Einführung für Ärzte und Studierende, 4. Auflage, Stuttgart, Georg Thieme Verlag, 1992

16. Roman S, Aladio P, Paris J, Nicollas R, Triglia JM. Prognostic factors of sudden hearing loss in children. Int J Padiatr Otorhinolaryngol 2001; 61: 17-21

17. Schmitt C, Patak M, Kröner-Herwig B. Stress and the onset of Sudden Hearing Loss and Tinnitus. Int Tinn Journ 2000; 6: 41-49

\section{Korrespondenzadresse:}

Priv.-Doz. Dr. med. Gerhard Goebel

Facharzt für Psychotherapeutische Medizin

Facharzt für Innere Medizin

Chefarzt der Medizinisch-Psychosomati-

schen Klinik Roseneck

D-83209 Prien am Chiemsee

E-Mail: ggoebel@schoen-kliniken.de 